\title{
Prevalence and genotypes of Enterocytozoon bieneusi in wildlife in Korea: a public health concern
}

Said Amer ${ }^{1,2}$, Sungryong Kim ${ }^{1}$, Jae-Ik $\mathrm{Han}^{3}$ and Ki-Jeong Na1,4*

\begin{abstract}
Background: Enterocytozoon bieneusi is a unicellular microsporidian fungal pathogen that infects a broad range of animal hosts, including wild and domestic animals and humans. The infection burden of this parasite in wild animals in Korea is largely unknown. In this study, the occurrence and genotypes of E. bieneusi were investigated in wild animal populations in Korea.

Methods: A total of 157 fecal samples ( 97 from Korean water deer, 48 from raccoon dogs and 12 from other taxa) were collected from wild animals at five wildlife centers in Korea. Genomic DNA was extracted from the samples and screened by nested-PCR targeting the internal transcribed spacer (ITS) region of rRNA, followed by sequence analysis to determine the genotype(s) of E. bieneusi.

Results: The overall prevalence of E. bieneusi was 45.2\% (71/157), with rates of 53.6\% (52/97) in Korean water deer, $35.4 \%(17 / 48)$ in raccoon dogs and $16.7 \%(2 / 12)$ in other taxa. We detected seven ITS genotypes, including one known (genotype D) and six new genotypes (Korea-WL1-Korea-WL6). Phylogenetically, all detected genotypes clustered with counterparts belonging to group 1, which includes isolates from different animal hosts and humans, suggesting their zoonotic potential.

Conclusions: Our survey results indicate that E. bieneusi circulates widely in wild animals in Korea. These findings address the role of wildlife as a potential source of microsporidiosis in domestic animals and humans.

Keywords: Enterocytozoon bieneusi, Prevalence, Wildlife, Korean water deer, Raccoon dog, Genotyping, South Korea
\end{abstract}

\section{Background}

Enterocytozoon bieneusi is a cosmopolitan microsporidian that infects a wide range of vertebrate and invertebrate hosts, including humans, domestic animals and wild game [1-6]. Enterocytozoon bieneusi infection is associated with enteropathy, resulting in diarrhea, malabsorption and occasionally growth impairment, especially in pediatric and immunocompromised individuals [712]. In addition, extra-intestinal microsporidian infections due to E. bieneusi are frequently reported [13-15]. Fecal-oral transmission is the most common route of

\footnotetext{
*Correspondence: sigol@cbnu.ac.kr

${ }^{1}$ Laboratory of Veterinary Laboratory Medicine and Wildlife Medicine, Veterinary Medical Center and College of Veterinary Medicine, Chungbuk National University, Cheongju, Chungbuk 28644, Republic of Korea Full list of author information is available at the end of the article
}

infection through spore-contaminated food and/or water $[9,16-18]$. Airborne transmission in cases of respiratory infections is still largely controversial [13]. Treatment options for E. bieneusi infections are limited, and few drugs have shown anti-microsporidian activity $[9,19]$.

Microscopic investigations of $E$. bieneusi spores in stool samples are inadequate to discriminate between genotypes owing to a lack of morphological differences. Molecular biology analyses are widely used for the sensitive detection and genotyping of microsporidian species $[20,21]$. The genetic structure of the internal transcribed spacer (ITS) region of rRNA shows high diversity among isolates [22], providing the ability to differentiate between host-adapted and zoonotic genotypes $[1,2,23]$ as well as the characterization of the geographical distribution of certain genotypes [24]. 
Enterocytozoon bieneusi is commonly detected in different wildlife, either in captive or free-living populations $[5,25-31]$, with ample range of genotypes of both hostadapted and those lacking host specificity $[1,32]$. Thus, wildlife animals are recognized as environmental reservoir for many human and animal infections [32, 33]. In Korea, a few studies have reported the molecular detection of E. bieneusi in livestock [34, 35] and in bats [36]; however, the infection burden of this microsporidian fungal pathogen in wildlife is largely unknown. Therefore, in the present study, the prevalence and genotypes of this parasite in wild animal populations in Korea were determined. Fecal samples were collected from wild animals at wildlife centers. Samples were screened for parasite occurrence by nested-PCR targeting the ITS region of rRNA. Genotypes were determined by sequence analysis of the positive samples. The relatedness of the obtained sequences was determined by alignment with reference sequences in GenBank and phylogenetic analysis.

\section{Methods}

\section{Collection of samples}

This study was performed from April to September, 2018, using samples collected from wild animals at five Korean wildlife centers (Fig. 1). A total of 157 fecal samples were collected from different animal taxa (Table 1), including 97 samples from Korean water deer (Hydropotes inermis argyropus), 48 from raccoon dogs (Nyctereutes procyonoides), 2 from roe deer (Capreolus pygargus), 3 from leopard cats (Prionailurus bengalensis), 3 from Eurasian otters (Lutra lutra), 3 from the Siberian weasel (Mustela sibirica) and 1 from a Eurasian badger (Meles meles). Fresh samples were placed in labeled plastic cups, mixed with 10 volumes of $70 \%$ ethanol, and stored at $4{ }^{\circ} \mathrm{C}$. The samples were transported in an ice box to the laboratory of Veterinary Laboratory Medicine at the Veterinary Medical Center, CNBU, Cheongju, Korea.

\section{DNA extraction and PCR amplification}

Ethanol was washed off the fecal samples with Milli Q water by centrifugation. Genomic DNA was extracted from $0.2 \mathrm{ml}$ of fecal slurry using a QIAamp DNA Stool Mini Kit (Qiagen, Hilden, Germany) according the manufacturer's instructions. DNA preparations were screened for E. bieneusi by nested PCR as previously described [5], with reagent grade water as a negative control. PCR was performed using 50- $\mu \mathrm{l}$ reaction mixtures consisting of $1 \times$ PCR buffer, $200 \mu \mathrm{M}$ dNTPs, $3 \mathrm{mM} \mathrm{MgCl}, 260 \mathrm{nM}$ primers, and 1.5 units of Taq DNA polymerase (Takara, Tokyo, Japan). Amplified fragments were electrophoresed on a $1.5 \%$ gel stained with EcoDye ${ }^{\mathrm{TM}}$ and visualized using UV light.

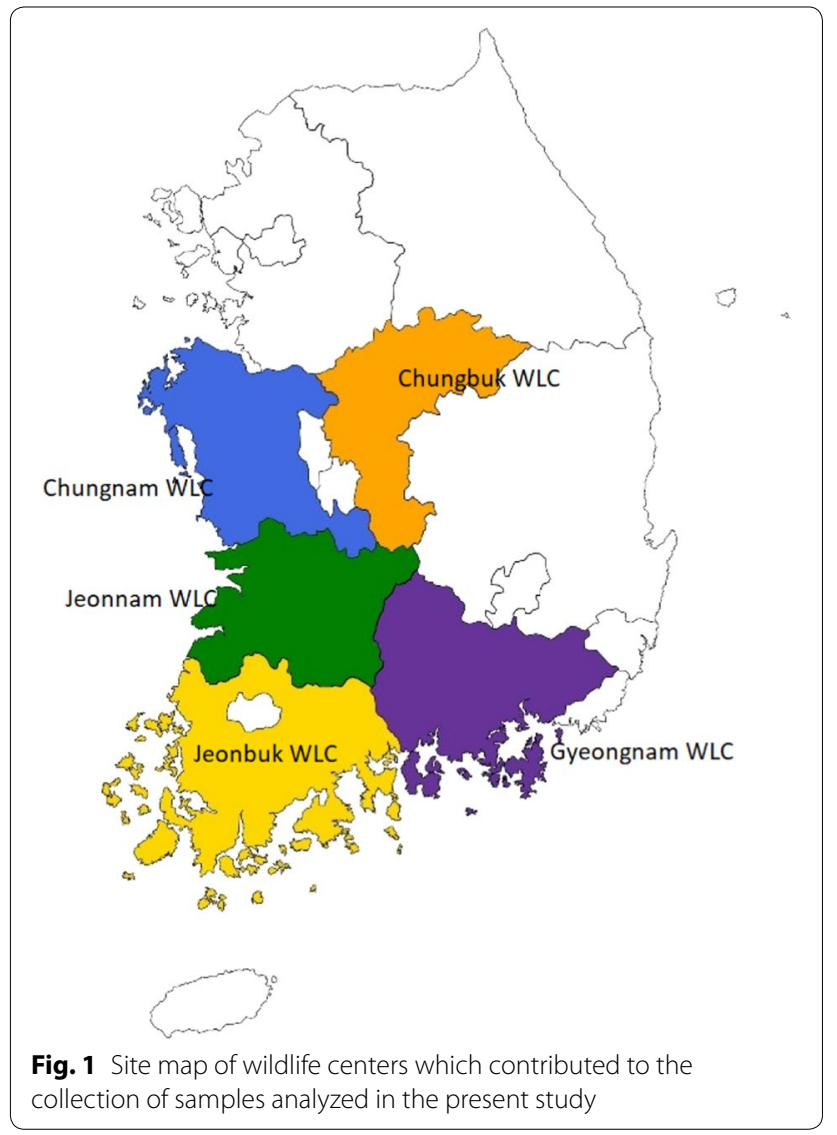

DNA sequence analysis

Secondary PCR products of positive samples were sequenced in both directions using Big Dye ${ }^{\circledR}$ Terminator v3.1 Cycle Sequencing Kit (Applied Biosystems, Foster City, CA, USA) and an ABI 3130 Genetic Analyzer (Applied Biosystems). Generated sequences were assembled using ChromasPro (https://techn elysium.com.au/wp/chromaspro/ v.2.1.8) and aligned with reference sequences in GenBank using ClustalX (http://www.clustal.org/) to determine the occurrence and genotype of E. bieneusi. Phylogenetic analysis was performed using maximum likelihood (ML) as implemented in MEGA v.7.0 (https://www.megasoftwa re.net/) with the Kimura 2-parameter model.

\section{Results}

\section{Occurrence of Enterocytozoon bieneusi}

Based on PCR and sequencing, we detected E. bieneusi in 71 out of 157 investigated samples, with an overall infection rate of $45.2 \%$. The prevalence was $53.6 \%$ $(52 / 97)$ and $35.4 \%(17 / 48)$ in Korean water deer and raccoon dogs, respectively (Table 1 ). In addition, one positive sample was detected in each of the roe deer 
Table 1 Distribution of samples and prevalence of E. bieneusi for each wildlife center and animal species

\begin{tabular}{|c|c|c|c|c|c|c|c|c|c|}
\hline \multirow[t]{2}{*}{ Location } & \multicolumn{2}{|c|}{$\begin{array}{l}\text { Korean water deer } \\
(n=97)\end{array}$} & \multicolumn{2}{|c|}{ Raccoon dog $(n=48)$} & \multicolumn{2}{|c|}{ Other taxa $(n=12)$} & \multirow[t]{2}{*}{ No. infected } & \multirow[t]{2}{*}{ Total number } & \multirow[t]{2}{*}{ Infection rate (\%) } \\
\hline & Infected & Uninfected & Infected & Uninfected & Infected & Uninfected & & & \\
\hline Chungbuk & 7 & 11 & 4 & 11 & 0 & 4 & 11 & 37 & 29.7 \\
\hline Jeonbuk & 16 & 12 & 13 & 7 & 1 & 2 & 30 & 51 & 58.8 \\
\hline ChungNam & 3 & 6 & 0 & 9 & 1 & 1 & 4 & 20 & 20.0 \\
\hline JeonNam & 3 & 7 & 0 & 4 & 0 & 3 & 3 & 17 & 17.7 \\
\hline GyungNam & 23 & 9 & 0 & 0 & 0 & 0 & 23 & 32 & 71.9 \\
\hline Total & 52 & 45 & 17 & 31 & 2 & 10 & 71 & 157 & $45.2^{\mathrm{a}}$ \\
\hline Infection rate (\%) & 53.6 & & 35.4 & & 16.7 & & & & \\
\hline
\end{tabular}

a Overall rate

Table 2 Positions of nucleotide changes within E. bieneusi genotype D-related sequences

\begin{tabular}{|c|c|c|c|c|c|c|c|c|c|c|}
\hline Genotype/sequence position & 152 & 159 & 160 & 174 & 232 & 259 & 277 & 298 & Host animal & Accession no. \\
\hline $\begin{array}{l}\text { Korea genotype D ( } 35 \\
\text { sequences)/MF693831 }\end{array}$ & G & $\mathrm{T}$ & G & G & G & G & G & A & $\mathrm{KWD}, \mathrm{RD}$ & LC436451-LC436482 \\
\hline Korea-WL1 (21 sequences) & . & . & & A & & . & . & . & KWD, $\mathrm{RD}$, roe deer & LC436483- LC436501 \\
\hline Korea-WL2 (11 sequences) & & & . & . & . & & $\mathrm{T}$ & & $\mathrm{KWD}, \mathrm{RD}$ & LC436502-LC436510 \\
\hline Korea-WL3 (1 sequence) & & & . & . & A & & & & $\mathrm{RD}$ & LC436511 \\
\hline Korea-WL4 (1 sequence) & A & & & & & . & $\mathrm{T}$ & . & LC & LC436512 \\
\hline Korea-WL5 (1 sequence) & . & - & - & & . & $\mathrm{T}$ & . & G & KWD & LC436513 \\
\hline
\end{tabular}

Abbreviations: KWD, Korean water deer; RD, raccoon dog; LC, leopard cat; -, deletion

$(1 / 2)$ and leopard cat (1/3). Samples from other taxa, including three otters, three weasels and one Eurasian badger, were negative. Infection was distributed in animals from all wildlife centers involved in the present study, with the highest infection rate detected at Gyungnam wildlife center (71.9\%), followed by Jeonbuk (58.8\%), Chungbuk (29.7\%), Chungnam (20.0\%) and Jeonnam (17.7\%).

\section{Enterocytozoon bieneusi genotypes}

Based on sequence analysis of the ITS gene marker, we detected seven genotypes, including one known (genotype D) and six novel genotypes (Korea-WL1-WL6). The known genotype $\mathrm{D}$ (detected in 35 samples isolated from 29 Korean water deer and 6 raccoon dogs) was identical to KX685189 from a human in Iran [11], KY950543 from a giant panda in China [28] and MF693831 from a sambar deer in Australia [5]. The novel genotypes KoreaWL1 (detected in 21 samples isolated from 12 Korean water deer, 8 raccoon dogs and 1 roe deer), Korea-WL2 genotype (detected in 11 samples isolated from 6 raccoon dogs and 5 Korean water deer) and Korea-WL3 (detected in one sequence isolated from a raccoon dog) showed one nucleotide substitution each at positions 174 (A/G), 277 (T/G) and 232 (A/G), respectively, compared to our genotype $\mathrm{D}$ sequences and the reference sequence (MF693831). We detected Korea-WL4 in one sequence (derived from sample of a leopard cat) with two substitutions (A/G and T/G) at positions 152 and 277, respectively. Korea-WL5, also detected in one sequence derived from a sample of Korean water deer, showed a 2-bp deletion at positions 152 and 153 as well as substitutions of T/G and G/A at positions 259 and 298, respectively, compared to genotype D (Table 2). Moreover, Korea-WL6 (generated from sample of a Korean water deer) showed a sequence identity of $98 \%$ with KR902354 detected in wastewater in China [37] and KP262358 detected from a goat in China [38], with seven nucleotide substitutions. Phylogenetically, all detected genotypes clustered with counterparts belonging to zoonotic group 1. Genotype D and novel genotypes Korea-WL1-WL5 clustered with other sequences of group 1a, whereas Korea-WL6 clustered with sequences in group 1e (Fig. 2).

\section{Discussion}

Our results provide the first description of the occurrence and genotypes of the microsporidian E. bieneusi in wild animal populations in Korea. We observed an overall prevalence of $45.2 \%$, with the highest infection rate $(53.6 \%)$ detected in samples from Korean water deer, 


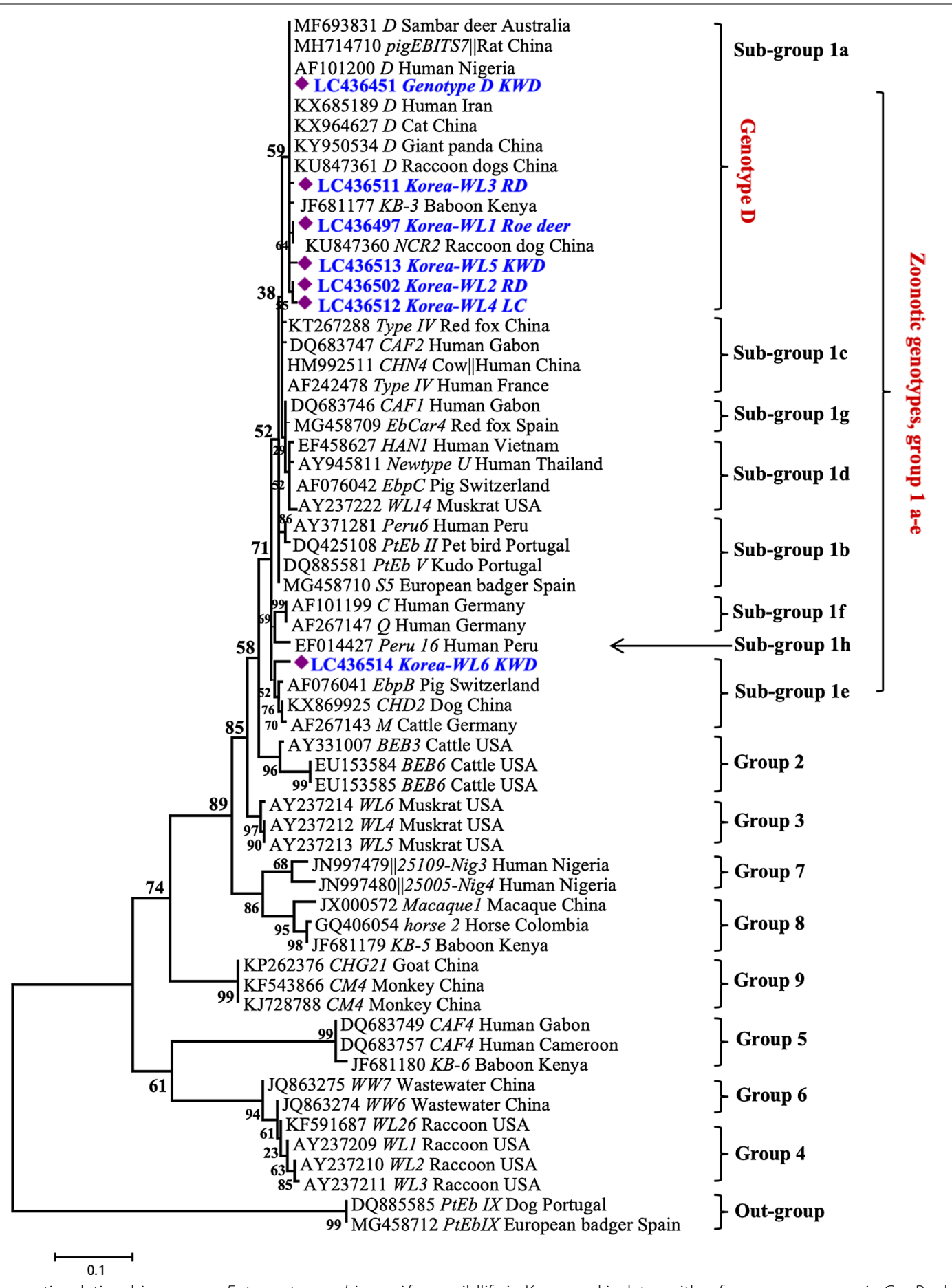

Fig. 2 Phylogenetic relationships among Enterocytozoon bieneusi from wildlife in Korea and isolates with reference sequences in GenBank. Evolutionary relationships were inferred based on internal transcribed spacer (ITS) sequences using the Maximum Likelihood (ML) method implemented in MEGA7. Branch support on the ML tree was calculated based on 1000 bootstrap replicates. Sequences obtained in this study are marked with diamonds on the tree. In all cases, the branch label includes accession number, followed by genotype name, host animal and country of origin. Abbreviations: KWD, Korean water deer; RD, raccoon dog; LC, leopard cat 
followed by raccoon dogs (35.4\%) and other taxa (16.7\%). Little is known about the occurrence of E. bieneusi in Korean water deer and free-range raccoon dogs. However, a low infection rate of $4.1 \%$ was recently reported in a survey of wild deer in water catchments in Melbourne, Australia [5], with all infections restricted to the sambar deer. The average E. bieneusi infection rates are $16.8 \%$ in wild reindeers [39], 34.0\% in wild Pere David's deer in China [40] and 32.5\% in wild white-tailed deer in the USA [41]. In farmed deer, the prevalence is $7-35 \%$ in sika deer, $20-37 \%$ in red deer [42-45] and $~ 17 \%$ in musk deer [31] in China. Compared with the $35.4 \%$ E. bieneusi infection rate in raccoon dogs in our study, a lower prevalence of $4-22 \%$ has been reported in farmed raccoon dogs (N. procyonoides) in China [29, 46, 47]. Moreover, rates of $E$. bieneusi occurrence differ according to the animal species and geographical region. Danišová et al. [48] reported an infection rate of $1.07 \%$ in wild mice in Slovakia, far lower than estimates of $38.9 \%$ in different wild rodents in Poland [25]. Additionally, a prevalence of $16.7 \%$ was observed in red-bellied tree squirrels in China [27]. A comparable prevalence of $41.2 \%$ was detected in Eurasian wild boars in China [28]. Similar rates of infection $(\sim 11 \%)$ have been observed in the herbivorous giant panda in China [26] and in wild carnivores ( 13\%) in Spain [33]. Humidity and temperature due to precipitation rate and vegetation types in forests in Korea are favorable environmental factors that maintain viability of E. bieneusi spores for long time and the social behavior of the Korean water deer and raccoon dogs that facilitates contact among families and groups of animals may be responsible for the high infection rate detected in the present study.

Our PCR and sequence analysis of the ITS region revealed a substantial degree of genetic diversity in the form of nucleotide substitutions and deletions. We identified seven distinct genotypes, including one known (genotype D) and six novel genotypes (Korea-WL1WL6). Comparable results for genetic diversity and new genotypes have been obtained for isolates from different species of wild and farmed deer in Australia and China $[5,39,40,44]$. Contrasting our findings, more new genotypes (up to 25) were detected in wild white-tailed deer in the USA [41] and in farmed sika deer in China [45], and only one or two new genotypes were detected in musk deer, red deer and Siberian roe deer in China [31, 43]. Genotype D of E. bieneusi detected in raccoon dogs in our study is a common genotype in farmed raccoon dogs in China [46], along with various (3-7) additional known and new genotypes [29, 46, 47]. Results of this study expand the geographical and host range of these genotypes.
Phylogenetic analysis (Fig. 2) revealed that E. bieneusi genotypes cluster in roughly eight groups. Genotypes of group 1 have no host specificity and were retrieved from different wildlife, domestic animals and humans, and those belonging to groups $2-8$ are almost all host-specific $[22,49,50]$. In this study, all detected genotypes were assigned to $E$. bieneusi group 1; 70 sequences belonged to $1 \mathrm{a}$ and one sequence belonged to 1e. Furthermore, isolates of genotype D, Korea-WL1 and Korea-WL2 reported in the present study were obtained from different hosts including Korean water deer, raccoon dogs and roe deer, indicating a lack of host specificity. However, the situation of host specificity for Korea-WL3 to KoreaWL6 genotypes is difficult due to isolation from only one sample. Similarly, most previously reported genotypes detected in wild and farmed deer and raccoon dogs belonged to group $1[5,29,39-41,45-47]$. The transmission of group 1 genotypes between animals and humans is likely, indicating that they have zoonotic potential [32, 51]. The exclusive identification of genotypes belonging to group 1 in this study emphasizes the role of wild populations as a source of environmental contamination; these genotypes are potentially hazardous for domestic animals and human health in Korea.

\section{Conclusions}

Our results provide the first evidence for the presence of E. bieneusi in wildlife in Korea. All infected animals carried potentially zoonotic pathogenic genotypes of this microsporidian. This study was limited by the uneven collection of samples from different wildlife centers and animal species, which my lead to biases in the results. Therefore, a larger study covering more geographical regions of Korea with even sample collection is underway to ascertain the role of wildlife as a reservoir for zoonotic gastrointestinal pathogen. The obtained results expand the geographical and host range of the detected genotypes and improve our understanding of the epidemiology of $E$. bieneusi infection in humans and animals in Korea.

\section{Abbreviations}

KWD: Korean water deer (Hydropotes inermis argyropus); ITS: internal transcribed spacer of nuclear ribosomal DNA.

\section{Acknowledgements}

The staff at contributing wildlife centers and College of Veterinary Medicine, Chungbuk National University are greatly appreciated.

\section{Funding}

This work was supported by the Brain Pool Programme funded by the Ministry of Science and ICT through the National Research Foundation of Korea (2018H1D3A2002236) 


\section{Availability of data and materials}

Nucleotide sequences generated in this study are available in the GenBank database under accession numbers LC436451-LC436514.

\section{Authors' contributions}

SA and KJN conceived of the study. SA, SK and JIH collected the samples and conducted the experiments. SA, JIH and KJN analyzed the data. SA and KJN prepared the report for publication. All authors read and approved the final manuscript.

\section{Ethics approval and consent to participate}

Not applicable.

\section{Consent for publication}

Not applicable.

\section{Competing interests}

The authors declare that they have no competing interests.

\section{Publisher's Note}

Springer Nature remains neutral with regard to jurisdictional claims in published maps and institutional affiliations.

\section{Author details \\ ${ }^{1}$ Laboratory of Veterinary Laboratory Medicine and Wildlife Medicine, Veteri- nary Medical Center and College of Veterinary Medicine, Chungbuk National University, Cheongju, Chungbuk 28644, Republic of Korea. ${ }^{2}$ Department of Zoology, Faculty of Science, Kafr El Sheikh University, Kafr El Sheikh 33516, Egypt. ${ }^{3}$ Laboratory of Wildlife Medicine/Diseases, College of Veterinary Medi- cine, Chonbuk National University, Iksan, Jeonbuk 54596, Republic of Korea. ${ }^{4}$ The Wildlife Center of Chungbuk, Cheongju, Chungbuk 28116, Republic of Korea.}

Received: 27 December 2018 Accepted: 2 April 2019

Published online: 08 April 2019

\section{References}

1. Sulaiman IM, Fayer R, Lal AA, Trout JM, Schaefer FW 3rd, Xiao L. Molecular characterization of microsporidia indicates that wild mammals harbor host-adapted Enterocytozoon spp. as well as human-pathogenic Enterocytozoon bieneusi. Appl Environ Microbiol. 2003;69:4495-501.

2. Sulaiman IM, Fayer R, Yang C, Santin M, Matos O, Xiao L. Molecular characterization of Enterocytozoon bieneusi in cattle indicates that only some isolates have zoonotic potential. Parasitol Res. 2004:92:328-34.

3. Didier ES, Weiss LM. Microsporidiosis: current status. Curr Opin Infect Dis. 2006;19:485-92

4. Chen D, Wang SS, Zou Y, Li Z, Xie SC, Shi LQ, et al. Prevalence and multi-locus genotypes of Enterocytozoon bieneusi in black-boned sheep and goats in Yunnan Province, southwestern China. Infect Genet Evol. 2018;65:385-91.

5. Zhang Y, Koehler AV, Wang T, Haydon SR, Gasser RB. First detection and genetic characterisation of Enterocytozoon bieneusi in wild deer in Melbourne's water catchments in Australia. Parasit Vectors. 2018;11:2.

6. Zou Y, Hou JL, Li FC, Zou FC, Lin RQ, Ma JG, et al. Prevalence and genotypes of Enterocytozoon bieneusi in pigs in southern China. Infect Genet Evol. 2018;66:52-6.

7. Kotler DP, Orenstein JM. Clinical syndromes associated with microsporidiosis. Adv Parasitol. 1998;40:321-49.

8. Tumwine JK, Kekitiinwa A, Bakeera-Kitaka S, Ndeezi G, Downing R, Feng $X$, et al. Cryptosporidiosis and microsporidiosis in Ugandan children with persistent diarrhea with and without concurrent infection with the human immunodeficiency virus. Am J Trop Med Hyg. 2005;73:921-5.

9. Anane S, Attouchi H. Microsporidiosis: epidemiology, clinical data and therapy. Gastroenterol Clin Biol. 2010;34:450-64.

10. Ghoshal U, Dey A, Ranjan P, Khanduja S, Agarwal V, Ghoshal UC. Identification of opportunistic enteric parasites among immunocompetent patients with diarrhoea from Northern India and genetic characterisation of Cryptosporidium and Microsporidia. Indian J Med Microbiol. 2016;34:60-6.

11. Ghoyounchi R, Ahmadpour E, Spotin A, Mahami-Oskouei M, Rezamand A, Aminisani N, et al. Microsporidiosis in Iran: a systematic review and metaanalysis. Asian Pac J Trop Med. 2017;10:341-50.

12. Hassan NA, Lim YAL, Mahmud R, Mohd-Shaharuddin N, Wan Sulaiman WY, Ngui R. Molecular diagnosis of microsporidia among immunocompromised patients in Kuala Lumpur, Malaysia. Am J Trop Med Hyg. 2018:99:1562-6.

13. del Aguila C, Lopez-Velez R, Fenoy S, Turrientes C, Cobo J, Navajas R, et al. Identification of Enterocytozoon bieneusi spores in respiratory samples from an AIDS patient with a 2-year history of intestinal microsporidiosis. J Clin Microbiol. 1997;35:1862-6.

14. Botterel F, Minozzi C, Vittecoq D, Bourée P. Pulmonary localization of Enterocytozoon bieneusi in an AIDS patient: case report and review. J Clin Microbiol. 2002:40:4800-1.

15. Kicia M, Sędzimirska M, Sak B, Kváč M, Wesołowska M, Hendrich AB, et al. Respiratory microsporidiosis caused by Enterocytozoon bieneusi in an HIV-negative hematopoietic stem cell transplant recipient. Int J Infect Dis. 2018:77:26-8.

16. Jedrzejewski S, GraczykTK, Slodkowicz-Kowalska A, Tamang L, Majewska AC. Quantitative assessment of contamination of fresh food produce of various retail types by human-virulent microsporidian spores. Appl Environ Microbiol. 2007;73:4071-3.

17. Decraene V, Lebbad M, Botero-Kleiven S, Gustavsson AM, Löfdahl M. First reported foodborne outbreak associated with microsporidia, Sweden, October 2009. Epidemiol Infect. 2012;140:519-27.

18. Galván AL, Magnet A, Izquierdo F, Fenoy S, Rueda C, Fernández Vadillo C, et al. Molecular characterization of human-pathogenic microsporidia and Cyclospora cayetanensis isolated from various water sources in Spain: a year-long longitudinal study. Appl Environ Microbiol. 2013;79:449-59.

19. Han B, Weiss LM. Therapeutic targets for the treatment of microsporidiosis in humans. Expert Opin Ther Targets. 2018;22:903-15.

20. Matos O, Lobo ML, Xiao L. Epidemiology of Enterocytozoon bieneusi infection in humans. J Parasitol Res. 2012;2012:981424.

21. Heyworth MF. Molecular diagnosis of human microsporidian infections. Trans R Soc Trop Med Hyg. 2017:111:382-3.

22. Santín M, Fayer R. Enterocytozoon bieneusi genotype nomenclature based on the internal transcribed spacer sequence: a consensus. J Eukaryot Microbiol. 2009;56:34-8.

23. Widmer G, Akiyoshi DE. Host-specific segregation of ribosomal nucleotide sequence diversity in the microsporidian Enterocytozoon bieneusi. Infect Genet Evol. 2010;10:122-8.

24. Henriques-Gil N, Haro M, Izquierdo F, Fenoy S, del Aguila C. Phylogenetic approach to the variability of the microsporidian Enterocytozoon bieneusi and its implications for inter- and intrahost transmission. Appl Environ Microbiol. 2010;76:3333-42.

25. Perec-Matysiak A, Buńkowska-Gawlik K, Kváč M, Sak B, Hildebrand J, Leśniańska K. Diversity of Enterocytozoon bieneusi genotypes among small rodents in southwestern Poland. Vet Parasitol. 2015;214:242-6.

26. Tian GR, Zhao GH, Du SZ, Hu XF, Wang HB, Zhang LX, et al. First report of Enterocytozoon bieneusi from giant pandas (Ailuropoda melanoleuca) and red pandas (Ailurus fulgens) in China. Infect Genet Evol. 2015:34:32-5.

27. Deng L, Li W, Yu X, Gong C, Liu X, Zhong Z, et al. First report of the human-pathogenic Enterocytozoon bieneusi from red-bellied tree squirrels (Callosciurus erythraeus) in Sichuan, China. PLoS One. 2016;11:e0163605.

28. Li W, Deng L, Wu K, Huang X, Song Y, Su H, et al. Presence of zoonotic Cryptosporidium scrofarum, Giardia duodenalis assemblage A and Enterocytozoon bieneusi genotypes in captive Eurasian wild boars (Sus scrofa) in China: potential for zoonotic transmission. Parasit Vectors. 2017:10:10.

29. Xu C, Ma X, Zhang H, Zhang XX, Zhao JP, Ba HX, et al. Prevalence, risk factors and molecular characterization of Enterocytozoon bieneusi in raccoon dogs (Nyctereutes procyonoides) in five provinces of northern China. Acta Trop. 2016;161:68-72

30. Cong W, Qin SY, Meng QF. Molecular characterization and new genotypes of Enterocytozoon bieneusi in minks (Neovison vison) in China. Parasite. 2018:25:34. 
31. Song Y, Li W, Liu H, Zhong Z, Luo Y, Wei Y, et al. First report of Giardia duodenalis and Enterocytozoon bieneusi in forest musk deer (Moschus berezovskii) in China. Parasit Vectors. 2018;11:204.

32. Leśniańska K, Perec-Matysiak A. Wildlife as an environmental reservoir of Enterocytozoon bieneusi (Microsporidia) - analyses of data based on molecular methods. Ann Parasitol. 2017;63:265-81.

33. Santín M, Calero-Bernal R, Carmena D, Mateo M, Balseiro A, Barral M, et al. Molecular characterization of Enterocytozoon bieneusi in wild carnivores in Spain. J Eukaryot Microbiol. 2018:65:468-74.

34. Jeong DK, Won GY, Park BK, Hur J, You JY, Kang SJ, et al. Occurrence and genotypic characteristics of Enterocytozoon bieneusi in pigs with diarrhea. Parasitol Res. 2007;102:123-8.

35. Lee JH. Prevalence and molecular characteristics of Enterocytozoon bieneusi in cattle in Korea. Parasitol Res. 2007;101:391-6.

36. Lee SH, Oem JK, Lee SM, Son K, Jo SD, Kwak D. Molecular detection of Enterocytozoon bieneusi from bats in South Korea. Med Mycol. 2018;56:1033-7.

37. Ma J, Feng Y, Hu Y, Villegas EN, Xiao L. Human infective potential of Cryptosporidium spp., Giardia duodenalis and Enterocytozoon bieneusi in urban wastewater treatment plant effluents. J Water Health. 2016:14:411-23.

38. Shi K, Li M, Wang X, Li J, Karim MR, Wang R, et al. Molecular survey of Enterocytozoon bieneusi in sheep and goats in China. Parasit Vectors. 2016;9:23.

39. Liu W, Nie C, Zhang L, Wang R, Liu A, Zhao W, Li H. First detection and genotyping of Enterocytozoon bieneusi in reindeers (Rangifer tarandus): a zoonotic potential of ITS genotypes. Parasit Vectors. 2015;8:526.

40. Zhang Z, Huang J, Karim MR, Zhao J, Dong H, Ai W, et al. Zoonotic Enterocytozoon bieneusi genotypes in Pere David's deer (Elaphurus davidianus) in Henan, China. Exp Parasitol. 2015;155:46-8.

41. Santín M, Fayer R. Enterocytozoon bieneusi, Giardia, and Cryptosporidium infecting white-tailed deer. J Eukaryot Microbiol. 2015:62:34-43.

42. Zhao W, Zhang W, Wang R, Liu W, Liu A, Yang D, et al. Enterocytozoon bieneusi in sika deer (Cervus nippon) and red deer (Cervus elaphus): deer specificity and zoonotic potential of ITS genotypes. Parasitol Res. 2014;113:4243-50.

43. Zhao W, Wang J, Yang Z, Liu A. Dominance of the Enterocytozoon bieneusi genotype BEB6 in red deer (Cervus elaphus) and Siberian roe deer (Capreolus pygargus) in China and a brief literature review. Parasite. 2017;24:54

44. Zhang XX, Cong W, Liu GH, Ni XT, Ma JG, Zheng WB, et al. Prevalence and genotypes of Enterocytozoon bieneusi in sika deer in Jilin Province, northeastern China. Acta Parasitol. 2016;61:382-8.

45. Huang J, Zhang Z, Yang Y, Wang R, Zhao J, Jian F, et al. New genotypes of Enterocytozoon bieneusi isolated from sika deer and red deer in China. Front Microbiol. 2017;8:879.

46. Yang Y, Lin Y, Li Q, Zhang S, Tao W, Wan Q, et al. Widespread presence of human-pathogenic Enterocytozoon bieneusi genotype $D$ in farmed foxes (Vulpes vulpes) and raccoon dogs (Nyctereutes procyonoides) in China: first identification and zoonotic concern. Parasitol Res. 2015;114:4341-8.

47. Zhao W, Zhang W, Yang Z, Liu A, Zhang L, Yang F, et al. Genotyping of Enterocytozoon bieneusi in farmed blue foxes (Alopex lagopus) and raccoon dogs (Nyctereutes procyonoides) in China. PLoS One. 2015;10:e0142611.

48. Danišová O, Valenčáková A, Stanko M, Luptáková L, Hasajová A. First report of Enterocytozoon bieneusi and Encephalitozoon intestinalis infection of wild mice in Slovakia. Ann Agric Environ Med. 2015;22:251-2.

49. Thellier M, Breton J. Enterocytozoon bieneusi in human and animals, focus on laboratory identification and molecular epidemiology. Parasite. 2008;15:349-58.

50. Karim MR, Dong H, Li T, Yu F, Li D, Zhang L, Li J, Wang R, Li S, Li X, Rume Fl, Ning C. Predomination and new genotypes of Enterocytozoon bieneusi in captive nonhuman primates in zoos in China: high genetic diversity and zoonotic significance. PLoS One. 2015;10:e0117991.

51. Kruse H, Kirkemo AM, Handeland K. Wildlife as source of zoonotic infections. Emerg Infect Dis. 2004;10:2067-72.
Ready to submit your research? Choose BMC and benefit from:

- fast, convenient online submission

- thorough peer review by experienced researchers in your field

- rapid publication on acceptance

- support for research data, including large and complex data types

- gold Open Access which fosters wider collaboration and increased citations

- maximum visibility for your research: over $100 \mathrm{M}$ website views per year

At BMC, research is always in progress.

Learn more biomedcentral.com/submissions 